\title{
AN ARCLENGTH PROBLEM FOR $m$-FOLD SYMMETRIC UNIVALENT FUNCTIONS
}

\author{
By SANFord S. Miller
}

1. Introduction. Let $S$ denote the class of functions

$$
f(z)=z+\sum_{n=2}^{\infty} a_{n} z^{n}
$$

which are analytic and univalent in the unit disk $\Delta:|z|<1$. Let $S^{*}$ denote the subclass of $S$ for which $f(z)$ is starlike, that is

$$
\operatorname{Re}\left[\frac{z f^{\prime}(z)}{f(z)}\right]>0 \quad(z \in \Delta) .
$$

Let $C$ denote the subclass of $S$ for which $f(z)$ is convex, that is

$$
\operatorname{Re}\left[\frac{z f^{\prime \prime}(z)}{f^{\prime}(z)}+1\right]>0 \quad(z \in \Delta) \text {. }
$$

Let $K$ denote the subclass of $S$ for which $f(z)$ is close-to-convex, that is

$$
\operatorname{Re}\left[\frac{z f^{\prime}(z)}{h(z)}\right]>0 \quad(z \in \Delta)
$$

where $h(z)$ is starlike. These classes are related by the proper inclusions $C \subset S^{*}$ $\subset K \subset S$.

A function $f(z)$ analytic in $\Delta$ is said to be $m$-fold symmetric $(m=1,2, \cdots)$ if

$$
f\left(e^{2 \pi i / m} z\right)=e^{2 \pi i / m} f(z) .
$$

In particular, every $f(z)$ is 1 -fold symmetric and every odd $f(z)$ is 2-fold symmetric. Let $S_{m}$ denote the subclass of $S$ for which $f(z)$ is $m$-fold symmetric. A simple argument shows that $f \in S_{m}$ is characterized by having a power series of the form

$$
f(z)=z+a_{m+1} z^{m+1}+a_{2 m+1} z^{2 m+1}+\cdots .
$$

We similarly define $S_{m}^{*}, C_{m}$ and $K_{m}$.

For $f \in S$ and $0<r<1$, let

$$
L_{r}(f)=\int_{|z|=r}\left|f^{\prime}(z)\right||d z|
$$

Received September 10, 1971. 
denote the arclength of the image of the circle $|z|=r$. Since $L_{r}(f)$ is a continuous functional and $S$ is a normal and compact family, a solution of the extremal problem

$$
\max _{f \in S} L_{r}(f)
$$

exists and is in the class $S$. The problem of finding the extremal function re mains unsolved. The problem

$$
\max _{f \in C_{1}} L_{r}(f)
$$

has been solved by Keogh [5] who showed that

The problem

$$
\max _{f \in C_{1}} L_{r}(f)=L_{r}\left(\frac{z}{1-z}\right)=\frac{2 \pi r}{1-r^{2}}
$$

$$
\max _{f \in S_{1}^{*}} L_{r}(f)
$$

has been solved by Marx [7] who showed that

$$
\max _{f \in S_{1}^{*}} L_{r}(f)=L_{r}(k)
$$

where $k(z)$ is the Koebe function

$$
k(z)=\frac{z}{(1-z)^{2}}=z+2 z^{2}+3 z^{3}+\cdots
$$

Clunie and Duren [1] have solved the extremal problem within the class $K$ and have shown that

$$
\max _{f \in K_{1}} L_{r}(f)=L_{r}(k)
$$

Duren [2] also obtained an evaluation of $L_{r}(k)$ in terms of standard elliptic integrals.

In $\S 2$ we deal with extending these results to $m$-fold symmetric functions $(m=1,2, \cdots)$ and solve the extremal problems

$$
\begin{array}{cl}
\text { I: } & \max _{f \in C_{m}} L_{r}(f), \\
\text { II: } & \max _{f \in S_{m}^{*}} L_{r}(f), \\
\text { III : } & \max _{f \in K_{m}} L_{r}(f),
\end{array}
$$

We shall need the following lemmas.

LEMMA 1. (a) $f(z) \in C_{m}$ if and only if $z f^{\prime}(z) \in S_{m}^{*}$;

(b) $f(z) \in S_{m}^{*}$ if and only if $\int_{0}^{z} \frac{f(\xi)}{\xi} d \xi \in C_{m}$. 
LEMMA 2. $f(z) \in S^{*}$ if and only if $\left[f\left(z^{m}\right)\right]^{1 / m} \in S_{m}^{*}$.

LEMMA 3. $f(z) \in S_{m}$ if and only if $f(z)=\left[g\left(z^{m}\right)\right]^{1 / m}$ where $g(z) \in S$.

LEMMA 4. $f(z) \in S_{m}^{*}$ if and only if

$$
f(z)=z \exp \int_{0}^{2 \pi} \ln \left(1-z^{m} e^{-i \phi}\right)^{-2 / m} d \mu(\phi)
$$

where $\mu(\phi)$ is non-decreasing on $[0,2 \pi]$ and $\mu(2 \pi)-\mu(0)=1$.

LEMma 5. Let $\mu(\phi)$ be non-decreasing on $[0,2 \pi]$ and $\mu(2 \pi)-\mu(0)=1$. If $h(\phi)$ is positive and integrable with respect to $\mu(\phi)$ on $[0,2 \pi]$ then

$$
\exp \int_{0}^{2 \pi} \ln h(\phi) d \mu(\phi) \leqq \int_{0}^{2 \pi} h(\phi) d(\phi) .
$$

LEMMA 6. If $f(z) \in K_{m}$ then

$$
f^{\prime}(z)=e^{i \alpha} \frac{g(z)}{z} P(z)
$$

where $g(z) \in S_{m}^{*}$, $\operatorname{Re} P(z)>0$ and

$$
P(z)=e^{-i \alpha}+b_{m} z^{m}+b_{2 m} z^{2 m}+\cdots .
$$

Lemma 1 is well known for the case $m=1$, Lemma 2 and Lemma 3 for $m=2$. The proofs in the general case are straightforward. Lemma 4 for the case $m=1$ may be found in $[2 ;$ p. 758] and the general case is easily handled using this result and Lemma 3. Lemma 5 is in [3; Thm. 208] while Lemma 6 may be found in [6].

\section{The arclength extremal problem.}

THEOREM 1. If $f \in C_{m}$, then $L_{r}(f) \leqq L_{r}\left(h_{m}\right)$ for $0<r<1$, where

$$
h_{m}(z)=\int_{0}^{z} \frac{\left[k\left(\xi^{m}\right)\right]^{1 / m}}{\xi} d \xi \in C_{m} .
$$

Proof. If $f \in C_{m}$, then by Lemma 1 (a) and Lemma 4 we have

$$
z f^{\prime}(z)=z \exp \int_{0}^{2 \pi} \ln \left(1-z^{m} e^{-i \phi}\right)^{-2 / m} d \mu(\phi),
$$

which yields

$$
\left|z f^{\prime}(z)\right|=|z| \exp \int_{0}^{2 \pi} \ln \left|1-z^{m} e^{-i \phi}\right|^{-2 / m} d \mu(\phi),
$$

where $\mu(\phi)$ is non-decreasing and $\mu(2 \pi)-\mu(0)=1$.

By Lemma 5 we obtain 


$$
\left|z f^{\prime}(z)\right| \leqq|z| \int_{0}^{2 \pi}\left|1-z^{m} e^{-i \phi}\right|^{-2 / m} d \mu(\phi)
$$

and consequently

$$
L_{r}(f)=\int_{0}^{2 \pi}\left|z f^{\prime}(z)\right| d \theta \leqq|z| \int_{0}^{2 \pi} \int_{0}^{2 \pi}\left|1-z^{m} e^{-i \phi}\right|^{-2 / m} d \mu(\phi) d \theta,
$$

where $z=r e^{i \theta}$. Changing the order of integration and using the identity,

$$
\int_{0}^{2 \pi}\left|1-z^{m} e^{-i \phi}\right|^{-2 / m} d \theta=\int_{0}^{2 \pi}\left|1-z^{m}\right|^{-2 / m} d \theta
$$

we obtain

$$
L_{r}(f) \leqq|z| \int_{0}^{2 \pi}\left|1-z^{m}\right|^{-2 / m} d \theta
$$

Since $k(z) \epsilon S^{*}$, by Lemma 2 we have $\left[k\left(z^{m}\right)\right]^{1 / m} \in S_{m}^{*}$, and by Lemma 1 (b),

$$
h_{m}(z) \equiv \int_{0}^{z} \frac{\left[k\left(\xi^{m}\right)\right]^{1 / m}}{\xi} d \xi \in C_{m} .
$$

A simple calculation yields

$$
L_{r}\left(h_{m}\right)=|z| \int_{0}^{2 \pi}\left|1-z^{m}\right|^{-2 / m} d \theta
$$

Hence (3) becomes $L_{r}(f) \leqq L_{r}\left(h_{m}\right)$ and we see that $h_{m}(z)$ is a solution of the ex tremal problem $\mathrm{I}$.

REMARKs. (i) For $m=1$

$$
h_{1}(z)=\frac{z}{1-z} \quad \text { and } \quad L_{r}\left(h_{1}\right)=\frac{2 \pi r}{1-r^{2}}
$$

(see Keogh [5]).

(ii) For $m=2$ (odd functions)

$$
h_{2}(z)=\frac{1}{2} \log \frac{1+z}{1-z}
$$

and

$$
L_{r}\left(h_{2}\right)=\int_{0}^{2 \pi} \frac{r}{\left|1-r^{2} e^{i 2 \theta}\right|} d \theta
$$

which can be expressed in terms of standard elliptic integrals.

(iii) For $m=3,4, \cdots$,

$$
h_{m}(z)=\int_{0}^{z} \frac{\left[k\left(\xi^{m}\right)\right]^{1 / m}}{\xi} d \xi=\int_{0}^{z} \frac{d \xi}{\left(1-\xi^{m}\right)^{2 / m}}
$$

maps $\Delta$ onto the interior of a regular convex polygon $P_{m}$ of order $m$ with peri- 
meter of length

$$
L_{m}=2^{1-4 / m} \frac{\Gamma^{2}\left(\frac{1}{2}-\frac{1}{m}\right)}{\Gamma\left(1-\frac{2}{m}\right)}
$$

[8; p. 196]. Since $h_{m}(z)$ maps $|z| \leqq r<1$ onto a convex subdomain of $P_{m}$ we must have $L_{r}\left(h_{m}\right) \leqq L_{m}$ for all $0<r<1$. Hence

$$
L_{r}(f) \leqq 2^{1-4 / m} \frac{\Gamma^{2}\left(\frac{1}{2}-\frac{1}{m}\right)}{\Gamma\left(1-\frac{2}{m}\right)}
$$

for $f \in C_{m}$, where $m=2,3, \cdots$ and $0<r<1$.

CoRollary. If $f \in C_{m}$ and $\rho(f ; r, \theta)$ is the radius of curvature of the image of $|z|=r(0<r<1)$ at $f\left(r e^{i \theta}\right)$ then

$$
\rho(f ; r, \theta) \leqq \frac{r\left(1+r^{m}\right)^{2-2 / m}}{1-r^{2 m}}
$$

and this bound is sharp.

Proof. The radius of curvature $\rho(f ; r, \theta)$ is given by

$$
\rho(f ; r, \theta)=\frac{\left|z f^{\prime}(z)\right|}{\operatorname{Re}\left[1+\frac{z f^{\prime \prime}(z)}{f^{\prime}(z)}\right]}
$$

[4; p. 359]. Using (1) we can show that

$$
\operatorname{Re}\left[1+\frac{z f^{\prime \prime}(z)}{f^{\prime}(z)}\right]=\int_{0}^{z} \frac{1-r^{2 m}}{\left|1-z^{m} e^{-i \phi}\right|^{2}} d \mu(\phi) .
$$

From (2) we have

$$
\begin{aligned}
\left|z f^{\prime}(z)\right| & \leqq r \int_{0}^{2 \pi}\left|1-z^{m} e^{-i \phi}\right|^{-2 / m} d \mu(\phi) \\
& =r \int_{0}^{2 \pi}\left|1-z^{m} e^{-\left.i \phi\right|^{-2}}\right| 1-\left.z^{m} e^{-i \phi}\right|^{2-2 / m} d \mu(\phi) \\
& \leqq r\left[\max _{\phi}\left|1-z^{m} e^{-\phi}\right|^{2-2 / m}\right] \int_{0}^{2 \pi}\left|1-z^{m} e^{-i \phi}\right|^{-2} d \mu(\phi),
\end{aligned}
$$

and consequently

$$
\left|z f^{\prime}(z)\right| \leqq r\left(1+r^{m}\right)^{2-2 / m} \int_{0}^{2 \pi}\left|1-z^{m} e^{-i \phi}\right|^{-2} d \mu(\phi) .
$$

From (4), (5) and (6) we obtain 


$$
\rho(f ; r, \theta) \leqq \frac{r\left(1+r^{m}\right)^{2-2 / m}}{1-r^{2 m}} .
$$

A simple calculation shows that

$$
\rho\left(h_{m} ; r, \pi / m\right)=\frac{r\left(1+r^{m}\right)^{2-2 / m}}{1-r^{2 m}},
$$

and since $h_{m} \in C_{m}$ we see that the bound in the corollary is sharp.

THeorem 2. If $f \in K_{m}$ then $L_{r}(f) \leqq L_{r}\left(\left[k\left(z^{m}\right)\right]^{1 / m}\right)$ for $0<r<1$.

Proof. Let

$$
\begin{aligned}
\mathscr{P}= & \{P(z) \text { regular in } \Delta \mid \operatorname{Re} P(z)>0, \\
& \left.|P(0)|=1, \text { and } P(z)-P(0)=b_{m} z^{m}+b_{2 m} z^{2 m}+\cdots\right\} .
\end{aligned}
$$

If $f \in K_{m}$, then by Lemma 6 we have

$$
z f^{\prime}(z)=e^{2 \alpha} g(z) P(z),
$$

where $g(z) \in S_{m}^{*}$ and $P(z) \in \mathscr{P}$. Since $g(z) \in S_{m}^{*}$, from Lemma 4 we obtain

$$
g(z)=z \exp \int_{0}^{2 \pi} \ln \left(1-z^{m} e^{-\imath \phi}\right)^{-2 / m} d \mu(\phi),
$$

where $\mu(\phi)$ is non-decreasing and $\mu(2 \pi)-\mu(0)=1$. Applying Lemma 5, we obtain the inequality

$$
|g(z)| \leqq|z| \int_{0}^{2 \pi}\left|1-z^{m} e^{-\imath \phi}\right|^{-2 / m} d \mu(\phi)
$$

Thus

$$
\left|z f^{\prime}(z)\right|=|g(z) P(z)| \leqq|z| \int_{0}^{2 \pi} \frac{d \mu(\phi)}{\left|1-z^{m} e^{-i \phi}\right|^{2 / m}}|P(z)|
$$

and for $z=r e^{i \theta}(0<r<1)$ we have

$$
L_{r}(f)=\int_{0}^{2 \pi}\left|z f^{\prime}(z)\right| d \theta \leqq \int_{0}^{2 \pi} \int_{0}^{2 \pi} \frac{|z| \cdot|P(z)|}{\left|1-z^{m} e^{-\imath \phi}\right|^{2 / m}} d \mu(\phi) d \theta
$$

On interchanging the order of integration and making the change of variable $\theta=\phi+\phi / m$, we obtain

$$
L_{r}(f) \leqq \int_{0}^{2 \pi} \frac{r\left|P\left(r e^{\imath(\psi+\phi / m)}\right)\right|}{\left|1-r^{m} e^{\imath m \psi}\right|^{2 / m}} d \psi d \mu(\phi) .
$$

If we let $\zeta=r e^{\imath \psi}$, then we obtain

$$
L_{r}(f) \leqq \int_{0}^{2 \pi} \int_{0}^{2 \pi} \frac{r\left|P\left(e^{2 \phi / m} \zeta\right)\right|}{\left|1-\zeta^{m}\right|^{2 / m}} d \phi d \mu(\phi)
$$




$$
\leqq \max _{0 \leqq \phi \leqq 2 \pi} \int_{0}^{2 \pi} \frac{r\left|P\left(e^{\imath \phi / m} \zeta\right)\right|}{\left|1-\zeta^{m}\right|^{2 / m}} d \phi .
$$

Since $P(\zeta) \in \mathscr{P}$, we have $P\left(e^{i \phi / m \zeta}\right) \in \mathscr{P}$ for $0 \leqq \phi \leqq 2 \pi$, and consequently

$$
L_{r}(f) \leqq \max _{P \in \mathcal{P}} \int_{0}^{2 \pi} \frac{r|P(\zeta)|}{\left|1-\zeta^{m}\right|^{2 / m}} d \psi .
$$

Now $P(\zeta)$ has a Herglotz representation given by

$$
\begin{aligned}
P(\zeta) & =\cos \alpha \int_{0}^{2 \pi} \frac{1+\zeta^{m} e^{-i t}}{1-\zeta^{m} e^{-i t}} d \nu(t)-i \sin \alpha \\
& =e^{-\imath \alpha} \int_{0}^{2 \pi} \frac{\left.1+\zeta^{m} e^{-\imath(t-2 \alpha}\right)}{1-\zeta^{m} e^{-i t}} d \nu(t),
\end{aligned}
$$

where $P(0)=e^{-\imath \alpha}, \nu(t)$ is non-decreasing and $\nu(2 \pi)-\nu(0)=1$. Thus

$$
|P(\zeta)| \leqq \int_{0}^{2 \pi} \frac{\left|1+\zeta^{m} e^{-\imath(t-2 \alpha)}\right|}{\left|1-\zeta^{m} e^{-i t}\right|} d \nu(t)
$$

and (7) can be replaced by

$$
\begin{aligned}
L_{r}(f) & \leqq \max _{\nu} \int_{0}^{2 \pi} \frac{r}{\left|1-\zeta^{m}\right|^{2 / m}} \int_{0}^{2 \pi} \frac{\left|1+\zeta^{m} e^{-\imath(t-2 \alpha)}\right|}{\left|1-\zeta^{m} e^{-i t}\right|} d \nu(t) d \psi \\
& =\max _{\nu} \int_{0}^{2 \pi} \int_{0}^{2 \pi} \frac{r}{\left|1-\zeta^{m}\right|^{2 / m}} \frac{\left|1+\zeta^{m} e^{-\imath(t-2 \alpha)}\right|}{\left|1-\zeta^{m} e^{-i t}\right|} d \psi d \nu(t) \\
& \leqq \max _{0 \leqq t \leqq 2 \pi} \int_{0}^{2 \pi} \frac{r}{\left|1-\zeta^{m}\right|^{2 / m}} \frac{\left|1+\zeta^{m} e^{-\imath(t-2 \alpha)}\right|}{\left|1-\zeta^{m} e^{-i t}\right|} d \psi .
\end{aligned}
$$

If we let $\phi=\theta+t / m$, we obtain

$$
\begin{aligned}
L_{r}(f) & \leqq r \max _{0 \leqq t \leqq 2 \pi} \int_{0}^{2 \pi} \frac{1}{\left|1-r^{m} e^{\imath(m \theta+t)}\right|^{2 / m}} \frac{\left|1+r^{m} e^{2(m \theta+2 \alpha)}\right|}{\left|1-r^{m} e^{2 m \theta}\right|} d \theta \\
& \equiv r \max _{0 \leqq t \leqq 2 \pi} I(\alpha, t) .
\end{aligned}
$$

It now follows from a result on rearrangements of functions given in $[1 ; \mathrm{p}$. 182] that

$$
I(\alpha, t) \leqq I(0,0) \text {. }
$$

Therefore

$$
L_{r}(f) \leqq \int_{0}^{2 \pi} \frac{r\left|1+r^{m} e^{\imath m \theta}\right|}{\left|1-r^{m} e^{2 m \theta}\right|^{2 / m+1}} d \theta=L_{r}\left(\left[k\left(z^{m}\right)\right]^{1 / m}\right) .
$$

Since $\left[k\left(z^{m}\right)\right]^{1 / m} \in S_{m}^{*}$ by Lemm 2, and $S_{m}^{*} \subset K_{m}$, we see from (8) that the function $\left[k\left(z^{m}\right)\right]^{1 / m}$ is a solution of the extremal problem III. This also indicates that it is a solution of problem II. 


\section{REFERENCES}

[1] Clunie, J., and P. L. Duren, Addendum: An arclength problem for close-toconvex function. Journal London Math. Soc. 41 (1966), 181-182.

[2] DuREn, P. L., An arclength problem for close-to-convex functions. Journal London Math. Soc. 39 (1964), 757-761.

[ 3 ] Hardy, G. H., J. E. Littlewood and G. Pólya, Inequalities. Second Edition, Cambridge University Press, London (1952).

[4] Hille, E., Analytic function theory, Vol. II. Blaisdell Publishing Co., Waltham, Mass.-Toronto-London (1962).

[5] Keogh, F. R., Some inequalities for convex and starshaped domains. Journal London Math. Soc. 29 (1954), 121-123.

[6] Maksimov, Y. D., Estimation of coefficients for certain classes of analytic functions. Dokl, Akad. Nauk SSSR (N.S.) 110 (1956), 507-510.

[7] MARX, A., Untersuchungen über schlichte Abbildungen. Math. Annalen 107 (1932), 40-67.

[8] Nehari, Z., Conformal mapping. McGraw-Hill Book Co., New York (1952).

Department of Mathematics,

State University of New York,

BROCKPORT, NEW YORK 14420

U.S.A. 